\title{
Medium-term scheduling of electric power system states under a wholesale electricity market
}

\author{
Natalia Aizenberg ${ }^{1}$, and Sergey Palamarchuk \\ Melentiev Energy Systems Institute SB RAS,Irkutsk, Russia
}

\begin{abstract}
The paper focuses on the development of a mathematical model for scheduling electric power system (EPS) states for the medium-term period divided into several time intervals. The model allows calculating the equilibrium state in the EPS, in which each supplier receives the maximum profit from the electricity supply to the wholesale market. The price levels in the EPS are determined by finding the maximum value of the social welfare given the balance constraints at the EPS nodes and the constraints on feasible state variables over several time intervals. Approaches to solving the multiinterval problem of search for an equilibrium states are considered. The approaches involve building a system of joint optimality conditions for electricity suppliers in the considered time intervals. The equilibrium state is found either by directly solving such a system or through an iterative search. The paper demonstrates the results of the medium-term scheduling of the state by an example of a simplified electric power system.. electric power system..
\end{abstract}

\section{Introduction}

Scheduling (forecasting) of states in electric power systems (EPSs) is an important task of operational dispatch control [1]. The states are scheduled for the short-term (for a coming day), medium-term (for a month, quarter, year), and long-term (for a period of up to 5 years) periods. The scheduling period is divided into time intervals (days, months, quarters).

In the medium-term scheduling, for all operating areas and all time intervals the System Operator determines [1]:

- electricity and power balances,

electrical connection diagrams and schedules of planned repairs of equipment at power plants; power lines and substations; relay protection devices; communication channels and control systems,

- state parameters to be maintained in time intervals of the scheduling period,

- transfer capabilities of electrical network cutsets, considering agreed repair schedules and meeting the requirements of reliability and power quality,

- types and volumes of services to ensure system reliability,

- activities in the case of planned and possible unplanned operating conditions of the power system.

The medium-term scheduling of EPS states takes into account:

- current and projected tariffs for electric and thermal energy,

- data on the results of trading in the wholesale electricity and capacity market (OREM), given the supply volumes stipulated in bilateral electricity purchase and sale agreements,

- characteristics of electrical networks, including transfer capabilities, losses, maximum allowable values of transmitted power,

- consumption rates of hydro resources for hydroelectric power plants (HPPs).

The load is distributed between the generating facilities according to the criterion of the minimum total costs for electricity buyers in the price zones of the wholesale electricity market. Traditionally, this aim was achieved by reducing the total costs associated with electricity production at thermal power plants. A larger-scale introduction of market relations in the electric power industry, a change in the structure of market participants and improvement in the rules of operational dispatch control have led to the modernization of the statements of scheduling problems and the change in the methods and algorithms for solving them [2]. New problem statements should take into account the facts that [3]:

- Electricity consumption in the medium term has price elasticity $[4,5]$. System operator should more carefully model the behavior of market prices and consider the possible reaction of consumers to their change;

- Most suppliers seek to maximize their profits. They do not aim at achieving the minimum total costs of electricity production in the electric power system; - Individual power plants are part of generating companies (GenCos) that pursue their corporate goals. Scheduling of the EPS states should not take into

\footnotetext{
${ }^{1}$ Corresponding author: ayzenberg.nata@gmail.com
} 
account the individual interests of power plants but rather their behavior as part of a generating company; - Individual suppliers can influence market prices. Electricity markets are markets with imperfect competition. Scheduling of the ESP state should allow for the oligopolistic nature of the wholesale market, in which the scheduling of generation by one supplier should take into account a possible behavior of other GenCos.

For the medium-term scheduling, one should consider the constraints that relate individual time intervals. This applies to the possibilities of changes in the generated power in each or several intervals, and to the constraints on the amount of electricity generated, energy resources consumed, and on the volume of stored energy [6].

This paper aims to introduce a new statement of the problem of medium-term scheduling of energy system states, which meets the conditions of the domestic wholesale electricity and capacity market and factors in the desire of electricity suppliers to achieve maximum profit. The focus is on the scheduling of the states in several time intervals given the inter-interval constraints and refinement of electricity prices at the nodes of its generation and consumption. The methods of solving the optimization problem are proposed and the EPS state scheduling results are demonstrated by a simplified example of a power system.

The first part of the paper discusses and formulates the problem statement. The second part describes possible approaches to balancing the interests of suppliers. One approach suggests building and solving a system of equations and inequalities consisting of optimality conditions for profit maximization by each supplier and social welfare maximization. The other approach uses an iterative method of approaching equilibrium. The third part of the paper is concerned with the numerical studies of the developed model capabilities. In addition, we analyze the qualitative differences in the calculation results depending on either presence or absence of inter-interval constraints and the type of the considered competition in the electricity market.

\section{Nomenclature}

The following notations are used in the text to describe the problem of medium-term scheduling of energy system states:

$t$ is number of time interval;

$T$ is the number of considered time intervals in a scheduling period;

$M$ is the number of time intervals with the interconnected state variables;

$\Delta t$ is the duration of time interval $t$;

$I_{n}^{t}$ is a set of numbers of nodes considered in time interval $t$;

$\mathfrak{R}_{i}^{t}$ is a set of numbers of nodes connected to node $i$;

$I_{g}^{t}$ is a set of numbers of nodes with generation;

$I_{d}^{t}$ is a set of numbers of nodes with electricity consumption;

$I_{f}$ is a set of numbers of nodes from $I_{g}^{t}$, whose power plants are part of the $f$-th GenCo;

$P_{g i}^{t}$ is the power generated at node $i$ in interval $t$,
$P_{\text {gimax }}^{t}$ is the maximum allowable value for $P_{g i}^{t}$;

$V_{g i}^{M}$ is the amount of generated electricity over $M$ intervals;

$V_{\text {gi max }}^{M}, V_{\text {gi min }}^{M}$ are the minimum and maximum amounts of generated electricity over $M$ intervals;

$P_{d i}^{t}$ is the power consumed at node $i$ in interval $t$;

$p_{i}^{t}$ is the electricity price at node $i$ in time interval $t$; $Q_{g i}^{t}$ is the water flow through the turbines of the $i$-th HPP in interval $t$;

$Q_{\text {gi } \max }^{M}, Q_{\text {gi min }}^{M}$ are the maximum and minimum allowable volumes of water drawdown from a reservoir of the $i$-th HPP or an allowable amount of fuel consumed at the $i$-th thermal power plant over $M$ intervals;

$P_{i j}^{t}$ is the power flow between nodes $i$ and $j$ in interval $t$;

$P_{i j \text { max }}^{t}$ is the maximum allowable value for $P_{i j}^{t}$;

$\Delta_{i j}^{t}$ is the share of losses from flow $P_{i j}^{t}$ in tie line $i-j$ in interval $t$;

$G K$ is a set of generating companies.

\section{A mathematical model of medium- term scheduling of EPS states}

Adequate representation of the electrical network and nodal prices is of great importance for scheduling of EPS states. In the practice of operational dispatch control, the consideration of the EPS steady states usually involves full nonlinear load flow models, including the equations of active and reactive power balances at the network nodes. Such models factor in the values of voltage magnitudes and phases, the transformation ratios of transformers, and the values of generated and consumed reactive power.

It is difficult to ensure a relatively accurate specification of such data in the medium-term scheduling in the time intervals that are remote from the time of modeling. The use of full non-linear power flow models with a large number of time intervals in complex power systems can prove too timeconsuming.

Simplified load flow modeling is justified for scheduling the expected EPS states. The simplified models factor in only active power distribution. Reactive power distribution is considered to be balanced and well known. In simplified models, power losses are most often represented as fractions of active power flows along the lines.

The proposed model of medium-term scheduling considers the simplified modeling of the steady states in EPS. The model is characterized by the following properties:

- electricity suppliers (GenCos) are interested in maximizing profits over the entire considered scheduling period [7],

- scheduling of the states allows attaining an equilibrium of the suppliers' interests. In the obtained state, GenCo does not seek to change the found values of the generating capacities $P_{g f}^{t}$, 
- scheduling takes into account the inter-interval constraints relating the state parameters of several considered time intervals. Such constraints affect the levels of locational market prices, increase the dimension of the optimization problem, and can significantly increase the complexity of solving it.

- scheduling of the states involves determining the values of nodal prices for electricity, which allows modeling a change in the consumed power $P_{d i}^{t}$ in each time interval $\mathrm{t}$.

The statement of the medium-term scheduling problem is as follows. Maximize the profit of individual generating companies over the entire scheduling period

$$
\begin{gathered}
S_{f}=\sum_{t}^{T} \sum_{i \in I f}\left(P_{g i}^{t} p_{i}^{t}-C_{i}^{t}\left(P_{g i}^{t}\right)\right) \rightarrow \\
\rightarrow \max , f \in G K,
\end{gathered}
$$

where $P_{g i}^{t} p_{i}^{t}$ is the revenue of the company from electricity sale in the amount of $P_{g i}^{t} \geq 0$ at prices $p_{i}^{t}$, $i \in I_{g}^{t}, \quad C_{i}^{t}\left(P_{g i}^{t}\right)$ is the function of costs for electricity production at node $i$.

Maximum of function (1) is determined following the constraints on the power flow values that provide:

Balances of active power at nodes of the network

$$
\begin{gathered}
P_{g i}^{t}-P_{d i}^{t}\left(p_{i}^{t}\right)-\sum_{j \in \Re \mathrm{i}}\left(P_{i j}^{t}-\left(1-\Delta_{j i}^{\mathrm{t}}\right) P_{j i}^{t}=0,\right. \\
i \in I_{n}^{t} .
\end{gathered}
$$

Bans on countercurrent power flow in one tie-line

$$
P_{i j}^{t} P_{j i}^{t}=0, \quad i \in I_{n}^{t}, j \in \mathfrak{R}_{\mathrm{i}}^{\mathrm{t}}, j>i .
$$

Constraints on power generation at a node with generation

$$
\begin{aligned}
& P_{g i \max }^{t} \geq P_{g i}^{t}, \quad i \in I_{g}^{t}, \\
& P_{g i}^{t} \geq P_{g i \min }^{t}, \quad i \in I_{g}^{t} .
\end{aligned}
$$

Constraints on power flow in tie-lines

$$
\begin{gathered}
P_{i j \text { max }}^{t} \geq P_{i j}^{t}, \quad i \in I_{n}^{t}, j \in \mathfrak{R}_{i}^{t}, j>i, \\
P_{i j}^{t} \geq P_{i j \min }^{t}, i \in I_{n}^{t}, j \in \mathfrak{R}_{i}^{t}, j>i, \\
P_{i j}^{t} \geq P_{j i}^{t}, \quad i \in I_{n}^{t}, j \in \mathfrak{R}_{i}^{t}, j>i, \\
P_{i j}^{t} \geq 0, \quad P_{j i}^{t} \geq 0, \quad i \in I_{n}^{t}, j \in \mathfrak{R}_{i}^{t}, j>i .
\end{gathered}
$$

Constraints (2) - (9) are considered for all intervals $t=1, \ldots, T$.

The system of equalities and inequalities (2) - (9) models steady state in the electric power system. Such a model factors in power losses in the network tieslines $\Delta_{i j}^{t}$ and does not require presetting the directions of flows $P_{i j}^{t}$ and $P_{j i}^{t}$. The sought variables in problem (1), (2) - (9) are generation volumes $P_{g i}^{t}, i \in I_{g}^{t}$, consumption volumes $P_{d i}^{t}, i \in I_{d}^{t}$, values of power flows $P_{i j}^{t}, P_{j i}^{t}, i \in I_{n}^{t}, j \in \Re_{i}^{t}, j>i$ in the tie-lines of the EPS equivalent circuit and nodal prices $p_{i}^{t}, i \in I_{n}^{t}$ in all intervals $t=1, \ldots, T$.

GenCos combine the individual power plants. The paper does not consider the case where one of the power system nodes has power plants that belong to different GenCos. We assume that suppliers (GenCos) behave independently in the market. Collusions between them and corporate behavior within the industrial groups are not considered in the paper. We assume that the price offers of suppliers correspond to their actual costs of electricity generation. The distortion of the presented price offers to increase company's profit is not taken into account in the paper.

The complexity of medium-term scheduling in a market environment stems from the need to simultaneously consider several time intervals. In this case, the inter-interval constraints relating the state parameters of several considered time intervals may participate in the optimization problem to be solved. Such constraints affect the levels of locational market prices, increase the dimension of the optimization problem, and can significantly increase the complexity of solving it.

In our research, we consider the following interinterval constraints:

- constraints on the amount of generated electricity $V_{g i}^{M}=\sum_{t \in M} P_{g i}^{t} \Delta t$ over $M$ intervals

$$
V_{g i \min }^{M} \leq V_{g i}^{M} \leq V_{g i \max }^{M}
$$

where $V_{g i \text { max }}^{M}, V_{g i \text { min }}^{M}$ are maximum and minimum allowable amounts of electricity generated over $M$ intervals at node $i$;

- constraints on the amount of energy resources consumed at node $i$ over $M$ intervals

$$
Q_{g i \min }^{M} \leq \sum_{t \in M} Q_{g i}^{t} \leq Q_{g i \max }^{M}
$$

Constraints (10), (11) are added to constraints (2) (9) in the statement of the medium-term scheduling problem. Other types of inter-interval constraints, namely, the constraints on the amount of energy stored in different types of storage systems and changes in the power generated over $M$ intervals are considered in [6].

Another difficulty of the medium-term scheduling is related to the dependence of consumption volumes $P_{d i}^{t}$ on the levels of nodal electricity prices. Demand for electricity for most consumers in the medium term has significant elasticity $[4,5]$. Therefore, System operator must take into account the reaction of wholesale consumers to changes in market prices. The medium-term scheduling should, where possible, allow for the consumer demand functions that accurately reflect their behavior when prices change at consumption nodes. 
The difficulty of considering the demand elasticity when scheduling power flows in a market environment arises because the dual variables to constraints (2) when solving the optimization problem with objective function (1) do not correspond to the values of nodal marginal prices for electricity in the considered power system. To determine the values of nodal prices, we need to solve an auxiliary optimization problem with an objective function that reflects the maximum social welfare. The auxiliary problem

$$
\begin{aligned}
W & =\sum_{t}^{T}\left(\sum_{i \in I_{d}} P_{d i}^{t} \cdot p_{i}^{t}\left(P_{d i}^{t}\right)-\sum_{i \in I_{g}} C_{i}^{t}\left(P_{g i}^{t}\right)\right) \rightarrow \\
& \rightarrow \max ,
\end{aligned}
$$

with constraints (2) - (9) is solved in each time interval $t$. In (12) $p_{i}^{t}\left(P_{d i}^{t}\right)$ is an inverse demand

function at node $i$ in interval $t$. Nodal prices $p_{i}^{t}$ correspond to dual variables $\lambda_{i}^{t}, i \in I_{n}^{t}$ to balance constraints (2) of the auxiliary problem (12), (2) - (9). The auxiliary problem is solved according to the technique of determining the hourly nodal prices, which is used by the Commercial operator of the domestic wholesale market [8].

For the medium-term scheduling, the auxiliary problem should factor in the inter-interval constraints (10), (11). The introduction of these constraints affects the levels of nodal prices for electricity $p_{i}^{t}$. Below, problem (12), (2) - (11) is called an extended auxiliary problem.

Summarizing the material of the section, it is worth noting that the medium-term scheduling of the power system states is reduced to simultaneously solving the extended auxiliary problem (12), (2) - (11) relative to the nodal prices $p_{i}^{t}$ and general scheduling problem (1), (2) - (11) that involves determining variables $P_{g i}^{t}, i \in I_{g}^{t}, \quad P_{d i}^{t}, i \in I_{d}^{t}, P_{i j}^{t}, P_{j i}^{t}, i \in I_{n}^{t}, j \in \Re_{i}^{t}, j>$ $i$ in all intervals $t=1, . ., T$.

One of the features of the medium-term scheduling problem is the lack of reliable information on the operation conditions for the power system in the upcoming time intervals. Apart from the unforeseen situations with the availability of the generation and network equipment, the uncertainty in the operation conditions is caused by changes in consumer bids for the volumes of electricity to be purchased from the market. It is difficult to predict the inflow of water into the reservoirs of the hydroelectric power plants and set the required levels of their lower pools several months in advance. Approximate information about the prospective levels of wholesale electricity prices reduces the quality of medium-term scheduling of power system states.

The lack of unambiguous data on the operation conditions requires the formulation of stochastic statements of the scheduling problem and the use of stochastic optimization methods. The development of such statements and methods is beyond the scope of the presented research and relates to the prospective line of our study. In this paper, the information on the operation of the wholesale market and the operation conditions of the energy system in the medium term is considered to be known and reliable.

\section{Methods for solving the problem of medium-term scheduling in a wholesale market environment}

One of the possible approaches to solving the above problem is the development of a mathematical model in the form of a system consisting of the KuhnTucker optimality conditions [9] for the following problems: a) profit maximization by suppliers (1), and b) extended auxiliary problem (12) with constraints (2) - (11).

Let us formulate the Kuhn-Tucker conditions for auxiliary problem (12), (2) - (11). Constraints (3) are considered for the case where the directions of flows are predetermined. We introduce the incidence matrix $E$ between the nodes with elements (13). If electricity flows from node $i$ to node $j$, the element $e_{i j}$ of matrix $E^{t}$ equals 1 , if it goes in a reverse direction, the element $e_{i j}$ of the matrix equals -1 , if there is no flow, $e_{i j}^{t}=0$.

$$
e_{i j}^{t}=\left\{\begin{array}{c}
1, \text { if } P_{i j}^{t} \geq 0 \\
-\left(1-\Delta_{j i}^{t}\right), \text { if } P_{j i}^{t} \geq 0 \\
0, \text { if } P_{i j}^{t}=0
\end{array}\right.
$$

Consider the inverse demand functions in each time interval at the nodes with electricity consumption to be specified in a linear form:

$$
p_{i}^{t}\left(P_{d i}^{t}\right)=h_{d i}^{t}-l_{d i}^{t} \cdot P_{d i}^{t}, \quad i \in I_{d}^{t}, t=1, \ldots, T .
$$

Assume that the cost function for electricity production for each supplier is quadratic:

$$
\begin{gathered}
C_{g i}^{t}\left(P_{g i}^{t}\right)=a_{g i}^{t}+b_{g i}^{t} \cdot P_{g i}^{t}+c_{g i}^{t} \cdot\left(P_{g i}^{t}\right)^{2}, \\
i \in I_{g}^{t}, \quad t=1, \ldots, T .
\end{gathered}
$$

The objective function of the auxiliary problem (12), given (14), has the form:

$$
\begin{aligned}
W= & \sum_{t}^{T}\left(\sum_{i \in I_{d}^{t}} P_{d i}^{t} \cdot\left(h_{i}^{t}-l_{i}^{t} \cdot P_{d i}^{t}\right)-\right. \\
& \left.\sum_{i \in I_{g}^{t}} C_{i}^{t}\left(P_{g i}^{t}\right)\right) \rightarrow \max ,
\end{aligned}
$$

where the first term in parentheses is demand $P_{d i}^{t}$ at node $i$, multiplied by price $p_{i}^{t}\left(P_{d i}^{t}\right)$. The objective function is concave with respect to variables $P_{d i}^{t}$ and $P_{g i}^{t}, \quad i \in I_{n}, t=1, \ldots, T$. Therefore, under the linear constraints (2) - (11), the problem has a unique solution [10].

Denote by $\lambda_{i}^{t}, \mu_{i}^{t}, \theta_{i j}^{t}, \gamma, \rho$ the dual variables for constraints (2) - (11). The Kuhn - Tucker optimality conditions [11] for the extended auxiliary problem (12), (2) - (11) are a mixed system of equalities and inequalities: 


$$
\begin{aligned}
& \lambda_{i}^{t} \cdot\left(P_{g i}^{t}-P_{d i}^{t}-\sum_{j \in \mathfrak{R}_{i}^{t}} e_{i j}^{t} \cdot P_{i j}^{t}\right)=0, \\
& i \in I_{n}, t=\overline{1, T} \text {; } \\
& \mu_{g i}^{t} \cdot\left(P_{g i \max }^{t}-P_{g i}^{t}\right)=0, \quad i \in I_{g}, \quad t=\overline{1, T} \\
& \theta_{i j}^{t}\left(P_{i j \max }^{t}-P_{i j}^{t}\right)=0, i \in I_{n}, j \in \Re_{i}^{t}, t=\overline{1, T} \\
& \gamma \cdot\left(V_{g i \max }^{M}-\sum_{t=1}^{M} P_{g i}^{t} \cdot \Delta t\right)=0, i \in I_{g}, \\
& i \in I_{n}, t=\overline{1, T} \text {; } \\
& \rho\left(Q_{g i \max }^{M}-\sum_{t=1}^{M} Q_{g i}^{t}\right)=0, i \in I_{g}, t=\overline{1, T} \\
& \left(P_{g i}^{t}-P_{g i \min }^{t}\right) \cdot\left(-\lambda_{i}^{t}-\gamma_{g i}^{1} \cdot \Delta t+\mu_{g i}^{t}+b_{g i}^{t}+\right. \\
& \left.+2 c_{g i}^{t} P_{g i}^{t}\right)=0, \quad i \in I_{g}, \quad t=\overline{1, T} \text {; } \\
& P_{d i}^{t}\left(\lambda_{i}^{t}-h_{d i}^{t}+2 l_{d i}^{t} \cdot P_{d i}^{t}\right)=0, i \in I_{d}, \quad t=\overline{1, T} \\
& \left(P_{i j}^{t}-P_{i j \min }^{t}\right)\left(\lambda_{i}^{t} \cdot e_{i j}^{t}+\theta_{i j}^{t}\right)=0, \\
& i \in I_{n}, j \in \mathfrak{R}_{i}^{t}, t=\overline{1, T} \text {; } \\
& P_{g i}^{t} \geq P_{g i \text { min }}^{t}, i \in I_{g}, t=\overline{1, T} \\
& P_{d i}^{t} \geq 0, \quad i \in I_{d}, t=\overline{1, T} \text {; } \\
& P_{i j}^{t} \geq P_{i j \text { min }}^{t}, \quad i \in I_{n}, j \in \mathfrak{R}_{i}^{t}, t=\overline{1, T} ; \\
& P_{g i \max }^{t}-P_{g i}^{t} \geq 0, \quad i \in I_{g}, \quad t=\overline{1, T} ; \\
& P_{i j \max }^{t}-P_{i j}^{t} \geq 0, i \in I_{n}, j \in \mathfrak{R}_{i}^{t}, t=\overline{1, T} \text {; } \\
& P_{g i \text { min }}^{t} \geq 0, P_{i j \text { min }}^{t} \geq 0, i \in I_{n}, j \in \mathfrak{R}_{i}^{t}, t=\overline{1, T} \text {; } \\
& V_{g i \max }^{M}-\sum_{t=1}^{M} P_{g i}^{t} \cdot \Delta t \geq 0, i \in I_{g}, \quad t=\overline{1, T} \\
& Q_{g i \max }^{M}-\sum_{t=1}^{M} Q_{g i}^{t} \geq 0, i \in I_{g}, \quad t=\overline{1, T} ; \\
& \mu_{g i}^{t} \geq 0, \quad i \in I_{g i}, \quad t=\overline{1, T} \\
& \theta_{i j}^{t} \geq 0, \quad i \in I_{n}, j \in \Re_{i}^{t}, t=\overline{1, T} \text {; } \\
& \gamma, \rho \geq 0 \text {. }
\end{aligned}
$$

By solving the system (15) - (33) we can obtain prices $p_{i}^{t}=\lambda_{i}^{t}$ at each node $i \in I_{n}$, for time intervals $t=1, \ldots, T$ of the scheduling period. These prices will be borne in mind by supplier $f$ when solving the problem of maximizing the total profit (1) for the entire scheduling period under conditions (15) - (33). To form optimality conditions for the general problem
(1), (2) - (11), it is necessary to supplement system (15)-(33) with the first-order conditions of function (1)

$$
\nabla S_{f}\left(P_{g_{f}}, \Lambda\right)=0, f \in G K,
$$

where $\nabla S_{f}(\cdot)$ is a gradient of function (1) for the $f$-th supplier, $\Lambda$ is a vector of dual variables $\lambda_{i}^{t}$. Then the Kuhn-Tucker optimality conditions for the general scheduling problem take the form of system (34), (15) - (33). Solving system (34), (15) - (33) ensures the combination of optimality conditions for the main problem (1), (2) - (11) and the extended auxiliary problem (12), (2) - (11).

The method of compiling system (34), (15) - (33) to determine the actions of suppliers is called the construction of a complementary model $[12,13]$. It can be used by generating companies to make offers for participation in auctions of the wholesale electricity market.

Compiling and solving system (34), (15) - (33) is a difficult task. Therefore, although the need for medium-term scheduling of the EPS states arose long ago, the algorithms for solving such problems are still under development $[14,15]$. This is due to some hindrances. The profit function of the supplier (1) has a bilinear term, i.e. revenue from the sale of electricity $P_{g i}^{t} \cdot p_{i}^{t}$ (here $p_{i}^{t}=\lambda_{i}^{t}$ ), which complicates solving problem (34), (15) - (33) and can lead to ambiguity of equilibrium solutions [16].

Also, the approach that involves the formation of joint optimality conditions requires solving cumbersome systems of equations/inequalities, which is difficult for the schemes of real electric power systems due to the large dimension and high computational effort of solving the problem (34), (15) - (33). For an electric power system with 100 nodes, 80 tie-lines, and 20 generation nodes integrated into 4 generating companies, scheduling of the states in 3 time intervals will require building and solving a system with more than 20,000 equations and inequalities.

The second possible approach to solving the problem of medium-term scheduling is to iteratively find an equilibrium of the supplier's interests. This is a fairly well-known approach $[17,18]$. Similarly to the previous approach, the Kuhn-Tucker system of conditions (15) - (33) is formed for an extended auxiliary problem. Further, at each iteration, one (not all) supplier solves the problem of profit maximization (1), considering the output of other players to be unchanged. After that, the found power $P_{g_{f}}$ of the $f$-th supplier is fixed. At the next iteration, another $k$-th company maximizes its profit knowing the generated power of the others. The procedure is repeated until all suppliers come to a state of equilibrium when none of them has an incentive to unilaterally change their generated power. The number of iterations in one calculation cycle is equal to the number of suppliers. The process of finding a solution consists of the following steps. 
1. Set the initial values of consumed power $P_{d i 0}^{t}, i \in$ $I_{d}$ in all time intervals.

2. By solving the problem (1), (2) - (11) for $t=$ $1, \ldots, T$, determine the initial values $P_{g i 0}^{t}, i \in I_{f}$ of all suppliers and values of the transmitted power $P_{i j 0}^{t}, i, j \in I_{n}$.

3. For one company $f_{1}$ determine the optimal values of the output in all time intervals by assuming that generated power of the other suppliers $f_{i} \neq f_{1}$ is known and fixed. To this end solve problem (34), (15) - (33), in which condition (34) is written only for $f_{1}$. After that fix the power generated at the nodes of company $f_{1}$ and consider the next supplier $k \neq 1$.

4. Solve problem (34), (15) - (33) for the $k$-th supplier. Fix the values of its generated power and switch to $(k+1)$-th supplier. After all $P_{g i}^{t}, i \in I_{g}^{t}$, $t=1, \ldots, T$ suppliers are found, go to the next cycle of calculations.

5. Repeat steps 3 and 4 until either the specified maximum number of calculation cycles is reached or an equilibrium solution is found. The latter can be defined as the EPS state, in which in the next cycle of calculations the previously found solution does not change or changes little.

Despite the fact that the equilibrium for the described model is theoretically not always unique $[13,17]$, for a wide range of characteristics of real power systems, we can expect that the equilibrium state will be found using the described iterative procedure [12].

\section{A numerical study of the mathematical model capabilities}

A numerical study was carried out on the example of a simplified power system with two suppliers and four power lines (Fig. 1). Section 3.1 presents the results for two options: a) maximization of social welfare, considering the inter-interval constraints (15)-(33), and $b$ ) optimization of social welfare, not considering the inter-interval constraints (15)-(28), (31), (32). For the maximizing social welfare, it is enough to calculate prices and volumes for the entire scheduling period only in the extended auxiliary problem. If provided from suppliers information is reliable, the obtained result will correspond to the maximum social welfare.

Section 3.2 discusses the effect of imperfect competition in the electricity market on the scheduling results. The option of scheduling a power system state under the imperfect competition involves the calculation of an equilibrium state in which each supplier is aimed to reach a maximum of its profit. For numerical study in section 3.2, the calculations are carried out using an iterative approach to solving problem (34), (15) - (33).

\section{Initial information.}

Figure 1 presents a simplified scheme of a power system. The scheduling period is assumed to consist of
3 time intervals $t=1,2,3$. Both suppliers in the system are thermal power plants.

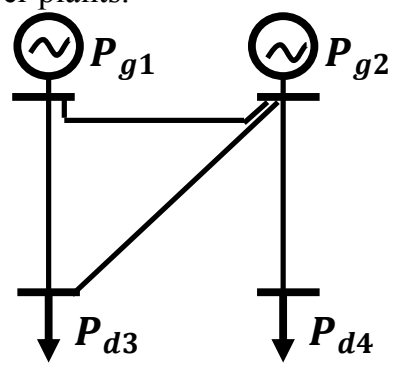

Fig.1. A scheme of the power system.

At nodes 1 and 2, there are generators with the same cost characteristics of electricity generation in all time intervals:

$$
\begin{gathered}
\mathrm{C}_{g 1}\left(P_{g 1}\right)=54200+72 P_{g 1}+4\left(P_{g 1}\right)^{2}, \\
C_{g 2}\left(P_{g 2)=} 21000+42.1 P_{g 2}+5.6\left(P_{g 2}\right)^{2} .\right.
\end{gathered}
$$

At nodes 3 and 4, electricity is consumed with demand characteristics that vary depending on the time interval:

$$
\text { For } t=1: \quad \begin{aligned}
P_{d 3}^{1} & =800-0.15 p_{3}^{1}, \\
& P_{d 4}^{1}=1600-0.38 p_{4}^{1} .
\end{aligned}
$$

$$
\text { For } \begin{aligned}
t=2,3: P_{d 3}^{2,3}= & 930-0.15 p_{3}^{2,3} \\
P_{d 4}^{2,3} & =1900-0.38 p_{4}^{2,3} .
\end{aligned}
$$

The fractions of power losses of flows in the lines are the same in all time intervals:

$\Delta_{1-2}=0.1, \Delta_{1-3}=0.12, \Delta_{2-3}=0.06, \Delta_{2-4}=0.08$.

Durations of time intervals in the considered example: $\Delta t^{1}=720, \Delta t^{2}=744, \Delta t^{3}=720$ hours.

Solving the problem of medium-term scheduling one can set integral constraints that relate state parameters of several time intervals. The considered example requires the fulfillment of the constraint on the total electricity output at node 2 for the intervals $t=1$ and $t=2$. The inter-interval constraint looks like $P_{g 2}^{1} \cdot \Delta t^{1}+P_{g 2}^{2} \cdot \Delta t^{2} \leq V_{g 2}^{1+2}$ max . If there is a hydroelectric power plant in the power system under consideration, the integral constraints can be set on the total volumes of water drawdown over several time intervals or the entire scheduling period.

\begin{tabular}{|c|c|c|c|c|c|c|}
\hline \multirow[t]{2}{*}{ Variable } & \multicolumn{3}{|c|}{$\begin{array}{l}\text { The minimum value in } \\
\text { the intervals }\end{array}$} & \multicolumn{3}{|c|}{$\begin{array}{l}\text { The maximum value in the } \\
\text { intervals }\end{array}$} \\
\hline & $t_{1}$ & $t_{2}$ & $t_{3}$ & $t_{1}$ & $t_{2}$ & $t_{3}$ \\
\hline$P_{g 1}$ & 20 & 20 & 40 & 120 & 140 & 180 \\
\hline$P_{g 2}$, & 0 & 40 & 40 & 280 & 320 & 320 \\
\hline$P_{1-2}$ & 40 & 40 & 10 & 250 & 270 & 300 \\
\hline$P_{1-3}$ & 10 & 10 & 10 & 120 & 125 & 140 \\
\hline
\end{tabular}

Table 1 shows the minimum and maximum allowable values of generated and transmitted electricity in all intervals of the considered scheduling period.

Table 1. Limiting values of variables. 


\begin{tabular}{|c|c|c|c|c|c|c|}
\hline $\boldsymbol{P}_{\mathbf{2}-\mathbf{3}}$ & 5 & 10 & 15 & 200 & 250 & 280 \\
\hline $\boldsymbol{P}_{\mathbf{2}-\mathbf{4}}$ & 0 & 10 & 15 & 200 & 250 & 260 \\
\hline
\end{tabular}

The maximum total electricity output at node 2 in the intervals $t=1$ and $t=2, V_{g 2}^{1+2}$ max $=416 \mathrm{GWh}$.

Table 2 shows the projected demand volumes. This is the reference information. If the optimal demand volumes obtained in the calculations differ significantly from the projected values, such an optimal option is rejected.

Table 2. Projected values of consumed power

\begin{tabular}{|c|c|c|c|}
\hline Parameter & \multicolumn{3}{|l|}{$\begin{array}{l}\text { Power values } \boldsymbol{P}_{\boldsymbol{d} \boldsymbol{i}}^{\boldsymbol{t}} \text { in the } \\
\text { intervals, } \mathbf{M W}\end{array}$} \\
\hline & $\boldsymbol{t}_{\mathbf{1}}$ & $\boldsymbol{t}_{\mathbf{2}}$ & $\boldsymbol{t}_{\mathbf{3}}$ \\
\hline $\boldsymbol{P}_{\boldsymbol{d} \mathbf{3}}^{\mathbf{1}}$ & 160 & 180 & 190 \\
\hline $\boldsymbol{P}_{\boldsymbol{d} \mathbf{4}}^{\mathbf{1}}$ & 180 & 220 & 230 \\
\hline
\end{tabular}

\subsection{Influence of inter-interval constraints on scheduling results}

The calculations are carried out and their results are compared with and without the inter-interval constraints (IIC).

Calculation 1. Scheduling of EPS states without the inter-interval constraint. The problem of maximizing social welfare

The calculation of the equilibrium without constraints (10), (11) entails solving the problem formulated using the Kuhn-Tucker conditions (15) (28), (31), (32). The search for a solution is carried out iteratively with the alternate fixing of the power generated by suppliers. The results are presented in Table 3.

Table 3. The results of the calculation of EPS states without inter-interval constraints while maximizing social welfare.

\begin{tabular}{|c|c|c|c|c|c|c|c|}
\hline $\begin{array}{l}\text { Vari } \\
\text { able }\end{array}$ & \multicolumn{2}{|l|}{$\begin{array}{l}\text { Values of variable, } \\
\text { MW in the } \\
\text { intervals }\end{array}$} & \multicolumn{4}{|l|}{$\begin{array}{l}\text { Prices at nodes, Rub/MW } \\
\text { in the intervals }\end{array}$} \\
\hline & $\boldsymbol{t}_{\mathbf{1}}$ & $\boldsymbol{t}_{\boldsymbol{2}}$ & $\boldsymbol{t}_{\mathbf{3}}$ & & $\boldsymbol{t}_{\mathbf{1}}$ & $\boldsymbol{t}_{\mathbf{2}}$ & $\boldsymbol{t}_{\mathbf{3}}$ \\
\hline $\boldsymbol{P}_{\boldsymbol{g} \mathbf{1}}$ & 120 & 140 & 180 & $\boldsymbol{p}_{\mathbf{1}}$ & 2786 & 3291 & 3179 \\
\hline $\boldsymbol{P}_{\boldsymbol{g} \mathbf{2}}$ & 262 & 310 & 299 & $\boldsymbol{p}_{\mathbf{2}}$ & 2976 & 3515 & 3395 \\
\hline $\boldsymbol{P}_{\boldsymbol{d} \mathbf{3}}$ & 162 & 184 & 194 & $\boldsymbol{p}_{\boldsymbol{3}}$ & 3166 & 3740 & 3612 \\
\hline $\boldsymbol{P}_{\boldsymbol{d} \mathbf{4}}$ & 184 & 223 & 239 & $\boldsymbol{p}_{\mathbf{4}}$ & 3242 & 3821 & 3741 \\
\hline \hline & \multicolumn{7}{|c|}{ Values of flows } \\
\hline $\boldsymbol{P}_{\mathbf{1 - 2}}$ & 40 & 40 & 40 & $\boldsymbol{P}_{\mathbf{2}-3}$ & 98 & 102 & 75 \\
\hline $\boldsymbol{P}_{\mathbf{1 - 3}}$ & 80 & 100 & 140 & $\boldsymbol{P}_{\mathbf{2}-\mathbf{4}}$ & 200 & 243 & 260 \\
\hline
\end{tabular}

Calculation 2. Scheduling with inter-interval constraint (10). The problem of maximizing social welfare.

The calculation of the equilibrium with constraint (10) entails solving the problem formulated using the Kuhn-Tucker conditions (15) - (29), (31) - (34). The calculation is given to show how inter-interval constraints can affect the results of the scheduling of states. In this example, an additional constraint (35) is imposed on the electricity generation of the second supplier at intervals 1 and 2. Table 4 presents the calculation results obtained by solving system (15) (33) for the entire scheduling period $T$.

Table 4. The results of the calculation of the EPS state with the inter-interval constraint for 3 time intervals while maximizing social welfare.

\begin{tabular}{|c|c|c|c|c|c|c|c|}
\hline $\begin{array}{c}\text { Variab } \\
\text { le }\end{array}$ & \multicolumn{3}{|c|}{$\begin{array}{c}\text { Values of } \\
\text { variables, } \\
\text { MW, in the } \\
\text { intervals }\end{array}$} & \multicolumn{6}{c|}{$\begin{array}{c}\text { Prices at nodes, } \\
\text { Rub/MW in the } \\
\text { intervals }\end{array}$} \\
\hline & $\boldsymbol{t}_{\mathbf{1}}$ & $\boldsymbol{t}_{\mathbf{2}}$ & $\boldsymbol{t}_{\mathbf{3}}$ & & $\boldsymbol{t}_{\mathbf{1}}$ & $\boldsymbol{t}_{\mathbf{2}}$ & $\boldsymbol{t}_{\mathbf{3}}$ \\
\hline $\boldsymbol{P}_{\boldsymbol{g} \mathbf{1}}$ & 120 & 140 & 180 & $\boldsymbol{p}_{\mathbf{1}}$ & 2814 & 3316 & 3179 \\
\hline $\boldsymbol{P}_{\boldsymbol{g} \mathbf{2}}$ & 254 & 302 & 299 & $\boldsymbol{p}_{\mathbf{2}}$ & 3006 & 3542 & 3395 \\
\hline $\boldsymbol{P}_{\boldsymbol{d} \mathbf{3}}$ & 160 & 182 & 194 & $\boldsymbol{p}_{\mathbf{3}}$ & 3198 & 3768 & 3612 \\
\hline $\boldsymbol{P}_{\boldsymbol{d} \mathbf{4}}$ & 179 & 219 & 239 & $\boldsymbol{p}_{\mathbf{4}}$ & 3268 & 3849 & 3741 \\
\hline \hline & \multicolumn{7}{|c|}{ Values of flows } \\
\hline $\boldsymbol{P}_{\mathbf{1 - 2}}$ & 40 & 40 & 40 & $\boldsymbol{P}_{\mathbf{2}-3}$ & 96 & 100 & 75 \\
\hline $\boldsymbol{P}_{\mathbf{1 - 3}}$ & 80 & 100 & 140 & $\boldsymbol{P}_{\mathbf{2}-\mathbf{4}}$ & 195 & 238 & 260 \\
\hline
\end{tabular}

A comparison of the results of Calculations 1 and 2 shows that when the inter-interval constraint (Table 4 ) is taken into account, the prices in the intervals $t=1$ and $t=2$ are higher than the corresponding prices in Table 3. In the time interval $t=3$, prices remained unchanged. In Calculation 2, the generated powers $P_{g 2}$ in the intervals $t=1$ and $t=2$ were reduced. Thus, the inter-interval constraints have a significant impact on the outcome of the medium-term scheduling of EPS states.

\subsection{Scheduling under imperfect competition}

Calculation 3. Scheduling of the EPS states with an inter-interval constraint. Imperfect competition.

In the conducted studies, the imperfect competition means the manifestation of oligopolistic properties of the electricity market. Scheduling of the electricity generation by individual suppliers aims to maximize profits given the possible behavior of other generating companies.

To search for a state that ensures a balance of interests of suppliers, we solved problem (34), (15) (33) using an iterative procedure based on successively solving problems (34), (15) - (33) for suppliers 1 and 2 . The results presented in Table 5 are obtained in 3 iterations.

Table 5. The results of the power system states scheduling with obtaining the equilibrium state

\begin{tabular}{|c|c|c|c|c|c|c|c|}
\hline \multirow[t]{2}{*}{\begin{tabular}{|c|}
$\begin{array}{c}\text { Varia } \\
\text { ble }\end{array}$ \\
\end{tabular}} & \multicolumn{3}{|c|}{$\begin{array}{l}\text { Values of variables, } \\
\text { MW in intervals } \\
\end{array}$} & \multicolumn{4}{|c|}{$\begin{array}{c}\text { Prices at nodes, Rub/MW } \\
\text { in intervals }\end{array}$} \\
\hline & $t_{1}$ & $t_{2}$ & $t_{3}$ & & $t_{1}$ & $t_{2}$ & $t_{3}$ \\
\hline$P_{g 1}$ & & 40 & 18 & 21 & & 220 & 2 \\
\hline$P_{g 2}$ & & 307 & 28 & & & & 35 \\
\hline$P_{d 3}$ & & & & & & & \\
\hline$P_{d 4}$ & & & 252 & & & 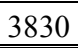 & 11 \\
\hline & \multicolumn{7}{|c|}{ of flows } \\
\hline$P_{1-2}$ & & & 0 & & & & 2 \\
\hline $\boldsymbol{P}_{1}$ & 80 & 100 & 140 & $P_{2-4}$ & 242 & 252 & 242 \\
\hline
\end{tabular}


The results of Calculation 3 show that under imperfect competition when the scheduling of states is reduced to finding a balance of interests of suppliers, the nodal prices for electricity (Table 5) increase in comparison with the prices of maximizing social welfare (Table 4). In the considered example, due to oligopolistic manifestations in the market, suppliers can raise prices by $2-4 \%$. This option provides the highest profit for suppliers and low amount of social welfare (Table 6). Consideration of such effects when scheduling medium-term states was the aim of this research.

Table 6. Supplier profits and social welfare amounts for the markets with different competition

\begin{tabular}{|c|c|c|c|}
\hline Profit & $\begin{array}{c}\text { Welfare } \\
\text { maximizatio } \\
\text { n without } \\
\text { IIC } \\
(15)-(28), \\
(31),(32) \\
\text { Calculation } \\
\mathbf{1}\end{array}$ & $\begin{array}{c}\text { Welfare } \\
\text { maximizatio } \\
\text { nith IIC } \\
\text { (15)-(33) } \\
\text { Calculation } \\
\mathbf{2}\end{array}$ & $\begin{array}{c}\text { Imperfect } \\
\text { competition } \\
\text { with IIC } \\
(34),(15)- \\
(33) \\
\text { Calculation } \\
\mathbf{3}\end{array}$ \\
\hline Supplier 1 & 907423 & 909755 & 918542 \\
\hline Supplier 2 & 1361999 & 1367369 & 1392410 \\
\hline $\begin{array}{c}\text { Social } \\
\text { welfare }\end{array}$ & 3808183 & 3806129 & 3800130 \\
\hline
\end{tabular}

\section{Conclusion}

The paper presents a mathematical model of medium-term scheduling of EPS states. The task is complicated by the need to allow for many timevarying factors and limitations. Changing EPS operation conditions include the balances of electricity and power, the implementation of repair schedules for generation and network equipment, the values of transfer capabilities of lines and cutsets, and the results of trading in the wholesale market.

Considering the properties of modern wholesale electricity markets, the formulated model factors in the interests of electricity suppliers that seek to maximize their profit in the case of medium-term scheduling. When scheduling, the constraints on the state parameters in each considered interval and interinterval constraints relating the state parameters in several time intervals are met.

The methods of solving the problem of mediumterm scheduling are considered. The methods include the formation of a complementary system of equalities and inequalities, which consists of Kuhn-Tucker optimality conditions for maximizing the social welfare and maximizing the profits of suppliers. Two methods for solving the formed problem are found. The first one seeks a solution to the entire complementary system, while simultaneously determining the equilibrium values of prices, output, demand, and transmission. The second one searches for a solution in an iterative way that converges to the sought state gradually, solving at each step the problem for one supplier only.

The numerical studies of the proposed mathematical model capabilities have been carried out using a simplified EPS as an example. For comparison, the calculations were performed for two problems: a) maximizing social welfare, and b) scheduling the states under an imperfect competition market. The numerical studies have confirmed the proposition that under imperfect competition, electricity suppliers can increase market price levels, and thus reduce the value of the social welfare.

\section{Acknowledgements.}

This work is carried out as part of the fundamental research program III.17.4.4 of the Siberian Branch of the Russian Academy of Sciences, scientific project № AAAA-A17-117030310433-6, and with the support of the Russian Foundation for Basic Research, grant № 019-010-00183.

\section{References}

1. The rules of operational dispatch control in the electric power industry. Gove. RF of 27.12.2004 N 854.

2. P.S. Abakshin Elect. Stat., 3 (2004

3. I.A.Nechaev, S.I. Palamarchuk, Proc. RAS. Power Engineering, 3 (2014)

4. A.V. Mishura, Region: Economics and Sociology, 2 (2009)

5. G. Shable, IEEE Power \& Energy, 9, 2 (2009)

6. T.A. Vaskovskaya, En. Syst. Research, 2, 6 (2019)

7. I.A. Nechaev, S.I. Palamarchuk, Proc. RAS. Power Engineering, 6, (2011)

8. The rules for competitive selection of price bids for the day ahead. Appendix 7

9. W.I. Zangwill Nonlinear programming. A unified approach, (1973)

10. Z.Q. Luo, J.S. Pang, D. Ralph Mathematical Programs with Equilibrium Constraints (1996)

11. A.V. Sokolov, V.V. Tokarev Methods for solving optimally, 1 (2011)

12. B.F. Hobbs, C.B. Metzler, J.S. Pang, IEEE Transact. Pow. Syst, 15, 2 (2000)

13. B.F. Hobbs, IEEE Transact. Pow. Syst, 16, 2 (2001)

14. S.A. Gabriel, F.U. Leuthold, Energy Econ, 32, 1 (2010)

15. L. Guo, G.H. Lin, D. Zhang, D. Zhu Oper. Res. Lett, 43, 3 (2015)

16. C.J. Day, B.F. Hobbs, J.S. Pang, IEEE Transact. Pow. Syst, 17, 3 (2002)

17. E. Bompard, W. Lu, R. Napoli, X. Jiang El. Pow. and En. Syst., 32 (2010)

18. J.D. Weber, T.J. Overbye, Proc. of IEEE PES Summer Meeting, 2 (1999) 